\title{
Association of single nucleotide polymorphism rs3792876 in SLC22A4 gene with autoimmune thyroid disease in a Chinese Han population
}

\author{
Xin Hou ${ }^{2,3}$, Jinyuan Mao ${ }^{2}$, Yushu Li ${ }^{1,2}$, Jia Li ${ }^{2}$, Weiwei Wang ${ }^{1,2}$, Chenling Fan², Hong Wang ${ }^{2}$, Hongmei Zhang ${ }^{2}$,
} Zhongyan Shan ${ }^{1,2}$ and Weiping Teng ${ }^{1,2^{*}}$

\begin{abstract}
Background: The autoimmune thyroid diseases (AITD), including Graves' disease (GD) and Hashimoto's thyroiditis (HT), are caused by interactions between susceptibility genes and environmental triggers. Single nucleotide polymorphisms (SNPs) of Solute carrier family 22, member 4 (SLC22A4) have been shown to be associated with several autoimmune diseases, including Crohn's disease (CD) and rheumatoid arthritis (RA). The aim of this study is to investigate whether SNP rs3792876 in the SLC22A4 gene is associated with GD, HT and AITD in a Chinese Han population.
\end{abstract}

Methods: In this study, we collected specimens from 553 Chinese Han individuals of 92 AlTD pedigrees in 10 cities in Liaoning province, China (80 GD pedigrees, 478 members; 12 HT pedigrees, 75 members). SNP rs3792876 was genotyped using the TaqMan allelic discrimination assay. Hardy-Weinberg Equilibrium tests were performed among founders of the pedigrees using Haploview software. Family-based association tests performed using FBAT software.

Results: No deviation from Hardy-Weinberg equilibrium was observed $(p>0.05)$. There were not significant association between the SLC22A4 gene polymorphism (rs3792876) and GD, HT and AITD was found.

Conclusions: These results suggest a lack of association between the SLC22A4 gene polymorphism rs3792876 and susceptibility to GD, HT and AITD in a Chinese Han population.

\section{Background}

The autoimmune thyroid diseases (AITDs) are common organ-specific autoimmune diseases with an estimated prevalence of $1.5 \%$ in general population [1]. They are characterized by infiltration of the thyroid with thyroid reactive lymphocytes and lead to two clinically opposing manifestations: Hashimoto's thyroiditis (HT) with hypothyroidism and Graves' disease (GD) affected by hyperthyroidism [2]. As most autoimmune disorders, AITD are complex conditions caused by interactions between genetic and environmental factors. Early family studies indicated that up to $50 \%$ of the siblings of patients

\footnotetext{
* Correspondence: twp@vip.163.com

'Department of Endocrinology and Metabolism, The First Hospital of China Medical University, Key Laboratory of Endocrine Diseases, Liaoning Province 110001 Shenyang, China

${ }^{2}$ Key Laboratory of Endocrine Diseases, Liaoning Province, Shenyang, China Full list of author information is available at the end of the article
}

with AITD were thyroid antibody positive in contrast to $15 \%$ in the general population [3], suggesting a strong genetic impact on the etiology of AITD. Since human leukocyte antigen (HLA) loci were first associated with GD [4], more than thirty chromosome regions [5] and a number of immune-related genes [6, 7] have been implicated in the genetic susceptibility to AITDs.

Among these genetic factors, the chromosome 5q31 region is of special interest because both our previous GD family study [8] and a Japanese AITD affected sib-pair study recognized 5q31 as an AITD susceptibility locus [9]. In addition, genetic variants in the $5 \mathrm{q} 31$ region have been shown to be responsible for autoimmune related disorders, such as Crohn's disease (CD) [3], atopic dermatitis (AD) [4] and bronchial asthma [5], by both classic genetic linkage and powerful genome-wide association (GWAS) studies. This chromosome region contains a cluster of 
cytokine and immune-related genes including IL4, IL13 and IRF1, suggesting a significant involvement of $5 \mathrm{q} 31$ in human cytokine and autoimmune regulation [10, 11]. However, limited studies have been carried out to further investigate the relationship between candidate genes in 5 q31 and AITDs, which makes it still unclear of the underlying mechanism for $5 \mathrm{q} 31$ genetic susceptibility to AITDs.

The SLC22A4 gene is located on chromosome $5 \mathrm{q} 31$ and encodes the organic cation transporter OCTN1. It is highly expressed in immune-related organs and tissues. SLC22A4 mRNA expression is induced by proinflammatory stimuli [12]. Genetic polymorphisms have been found to affect SLC22A4 gene expression and to be associated with autoimmune diseases such as rheumatoid arthritis (RA),CD and type 1 diabetes (T1D) [12-14]. All these findings suggest the essential role of $S L C 22 A 4$ in the autoimmune regulatory pathway. It maybe related to autoimmune diseases in the development of autoimmunity in secondary lymphoid organs [12]. Studies of an intron 1 single nucleotide polymorphism (SNP) rs3792876 in SLC22A4 showed that this polymorphism affected binding of a transcription regulator, runt-related transcription factor 1 (RUNX-1) $[15,16]$ and might regulate the transcription of SLC22A4 [12]. Several other recent publications have revealed that polymorphisms affecting RUNX-1 binding sites may be associated with a variety of autoimmune diseases such as psoriasis [15] and systemic lupus erythematosus (SLE) [16]. Despite all these evidences of involvement of SLC22A4 in human autoimmune disease, the association of SLC22A4 with AITD has never been studied. We hypothesized that SLC22A4 might be involved in genetic susceptibility of AITD through the transportation of small organic molecules (such as immune regulatory factors), and such involvement might be mediated by a SNP located in a RUNX1-binding sequence in SLC22A4 affecting the expression of $S L C 22 A 4$. In this study we collected AITD pedigrees (GD pedigrees and HT pedigrees), genotyped rs3792876 and assess the genetic susceptibility of this SLC22A4 polymorphism to GD, HT and AITD in our Chinese Han populations.

\section{Methods}

\section{Subjects ascertainment}

In this study 92 Chinese Han AITD pedigrees (80 GD pedigrees and $12 \mathrm{HT}$ pedigrees) with 553 individuals were recruited in Liaoning Province, China. Pedigrees were ascertained through GD or HT probands at the outpatient clinic of Department of Endocrine Diseases of the First Hospital of China Medical University. Family history was subsequently confirmed by at least one affected first-degree family member. HT was diagnosed as follows: 1) medical history or clinical biochemistry evidence of hypothyroidism requiring thyroid hormone replacement therapy, and 2) presence of autoantibodies to thyroidperoxidase, with or without antibodies to thyroglobulin. GD was diagnosed as follows: 1) medical history or clinical biochemistry evidence of hyperthyroidism, 2) diffused goiter, and 3) the presence of at least one of the following manifestations: positive thyroid stimulating hormone (TSH) receptor antibody tests, diffusely increased ${ }^{131} \mathrm{I}$ (iodine-131) uptake in the thyroid gland or presence of exophthalmos. All other family members were classified as unaffected, whether their thyroid autoantibody tests were positive or negative. All diagnoses were made by at least two independent endocrine physicians. Research protocols were approved by the medical ethics committee of the China Medical University and conformed to the guidelines set forth by the Declaration of Helsinki. Written informed consents were acquired from all of the participants or their guardians. Peripheral venous blood samples were collected from all of the participants for DNA preparations and biochemical measurements.

\section{Genotyping}

Genomic DNA was isolated from peripheral white blood cells using the DNA purification kit (QIAgen, Hilden, Germany) according to the manufacturer's instructions. The rs3792876 SNP was genotyped by Taqman allelic discrimination assay on a Roche LC480 Real-Time PCR System (Roche Diagnostics, Penzberg, Germany). The SNP was genotyped using a predesigned TaqMan SNP Genotyping Assay (Applied Biosystems; assay ID C_3170428_10). Amplification was performed in a total of 10ul containing $30 \mathrm{ng}$ of template DNA according to the manufacturer's standard program protocol. $5 \%$ of the samples were randomly chosen to confirm the genotypes by direct sequencing for quality control.

\section{Statistical methods}

The Hardy-Weinberg Equilibrium was test among founders using Haploview4.2 [17]. Family-based association tests (FBAT) were performed using the FBAT software [18] (V2.0.3 Harvard School of Public Health, Departments of Biostatistics and Environmental Health, Program for Population Genetics, Boston, Massachusetts, USA). All of the tests were performed under the assumption of an additive model, with a null hypothesis of no linkage and no association. A $P<0.05$ was considered statistically significant. Differences in genotype frequencies between patients and cases were calculated by chi-square or Fisher's exact test when necessary.

\section{Result}

Among 92 AITD pedigrees (553 individuals), 29 families (32\%) had2patients, 39 (42\%) had 3and17 (18\%) had 4, 
5 (5 \%) had 5 and 2 (2\%) had 6. Among the 80 GD pedigrees (478 individuals), 20 (25\%) had 1affected firstdegree relatives, 37 (46\%) had 2, 16 (20\%) had 3, 5 (6\%) had 4 and $2(3 \%)$ had 5. In total, there were 201 GD patients (45males and156 females; male/female ratio of 1:3.47; mean age of $43.72 \pm 14.31$ years) and 277 firstdegree relatives (136 males and141 females, mean age of $48.95 \pm 16.98$ years). Among the $12 \mathrm{HT}$ pedigrees (75 individuals), $9(75 \%)$ had 1affected first-degree relatives, $2(17 \%)$ had 2 , and $1(8 \%)$ had 3. There were $29 \mathrm{HT}$ patients (8 males and 21 females, male/female ratio of 1:2.62, mean age of $42.45 \pm 19.85$ years) and 46 firstdegree relatives (18 males and 28 females, mean age of $47.55 \pm 16.75$ years). The patients' general condition was list in Table 1.

No deviation from Hardy-Weinberg equilibrium was detected among all founders $(P>0.05)$. The genotyping call rate was $99.6 \%$ and the sequencing confirmation rate was $100 \%$. In total, $80 \mathrm{GD}, 12 \mathrm{HT}$ and $92 \mathrm{AITD}$ pedigrees were genotyped for the SNP rs3792876. The T allele frequency of rs3792876 was $21 \%$ in GD pedigrees and $20 \%$ in AITD pedigrees. In addition, we performed a FBAT; however, there was no significant association detected $(p>0.05)$ (Table 2). We also compared the genotype of rs3792876 in GD, HT and AITD patients and controls, there was no significant association $(p>0.05)$ (Table 3).

\section{Discussion}

Our previous study mapped a susceptible locus of GD in chromosomal 5q31 regions [8]. A maximum LOD score over 4 indicated a strong linkage between a GD phenotype and polymorphic genetic markers in this region. We focused on this region in a subsequent study because this region contains many immune related genes associated with autoimmune disorders or traits such as $\mathrm{CD}$, asthma, arthritis and T1D. We tested the linkage between SNPs in 5q31 genes IL4, IL13, IRF1 and UGRP1 in Chinese GD patients [19]. However, a negative association was observed.
Table 2 FBAT analysis of the association between GD, HT and AITD and rs37928

\begin{tabular}{llllll}
\hline Disease & Family & S-E(S) & $\operatorname{Var}(\mathrm{S})$ & $Z$ & $P$ \\
\hline GD & 39 & 1.40 & 34.91 & 0.24 & 0.813 \\
HT & 1 & - & - & - & - \\
AITD & 40 & 0.65 & 35.47 & 0.11 & 0.913 \\
\hline $\begin{array}{l}\text { Family: Number of families that are actually informative for the test. S: test } \\
\text { statistics for the observed number of transmitted alleles. E(S): expected value } \\
\text { of S under the null hypothesis (i.e., no linkage or association) }\end{array}$
\end{tabular}

In this study, we chose $S L C 22 A 4$ as candidate gene based on current knowledge on its molecular function and genetic predisposition studies. SLC22A4 encodes an organic cation transporter mainly expressed in the liver, kidney, intestine and other organs. It is critical for the elimination of many endogenous small organic cations as well as a wide array of drugs and environmental toxins. A functional intronic polymorphism rs3792876 has been studied in several different ethnic groups for an association with immune disorders including CD, T1D and RA. However, inconsistent results have been obtained. The association of rs3792876 with RA was originally reported in a Japanese population. The researchers suggested an allelic difference of rs3792876 in affinity to RUNX1, a transcriptional regulator in the hematopoietic system [12]. This association was replicated in other Japanese populations $[20,21]$ but not in Caucasian and Spanish groups $[22,23]$. The SNP rs3792876 was also shown to genetically predispose patients to T1D [13] and CD [24]. However, these findings were never confirmed.

Before the current study, the association between rs3792876 and GD, HT and AITD has never been assessed. Our negative results may reflect the real condition that this variant may confer to limit or even no contribution to etiology of GD, HT, and AITD. Although previous studies suggested rs3792876's involvement into immune disorders, due to pathophysiological difference between AITD and other autoimmune diseases, it's highly possible that $S L C 22 A 4$ may be genetically totally unrelated to GD, HT, or AITD.

Table 1 The clinical manifestation and biochemical characterization of GD, HT and AITD

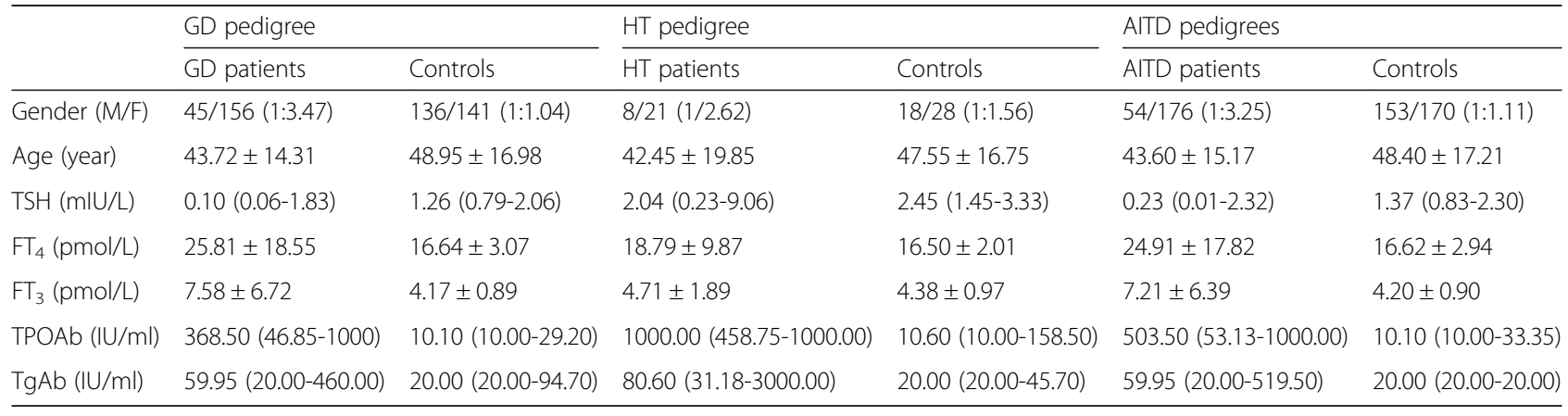


Table 3 Comparison of genotype of rs3792876 in GD, HT and AITD patients and controls

\begin{tabular}{|c|c|c|c|c|c|c|}
\hline & \multicolumn{2}{|c|}{ GD pedigree } & \multicolumn{2}{|c|}{ HT pedigree } & \multicolumn{2}{|c|}{ AITD pedigree } \\
\hline & $\begin{array}{l}\mathrm{GD} \\
\text { patients }\end{array}$ & Controls & $\begin{array}{l}\mathrm{HT} \\
\text { patients }\end{array}$ & Controls & $\begin{array}{l}\text { AlTD } \\
\text { patients }\end{array}$ & Controls \\
\hline CC & 123 & 180 & 23 & 35 & 146 & 215 \\
\hline$C T$ & 70 & 85 & 6 & 9 & 76 & 94 \\
\hline$\pi$ & 8 & 12 & 0 & 2 & 8 & 14 \\
\hline P & 0.633 & 0.532 & 0.571 & & & \\
\hline
\end{tabular}

Potential limitations of this study must also be addressed. First, the sample size of our study is relatively small, which may not have the efficient statistical power to detect a weak genetic effect, resulting in a fluctuated estimation. Second, we evaluated association of only one common polymorphism of SLC22A4 gene with susceptibility to GD, HT and AITD, there might be still other unidentified SNPs which could influence the development of GD, HT or AITD. Third, our pedigrees are all Han Chinese. Genetic background is a very important issue in genetic association study and may affect both association strength and direction. Rs3792876 allelle frequencies differ greatly among different ethnic groups with highest MAF in East Asian $(>0.2)$ and much lower in other populations $(<0.1)$. Therefore, more studies with larger sample size, multiple ethnic groups and more candidate genes are needed to further study the molecular genetic basis underlying chromosome region 5 p31 in predisposition to GD, HT and AITD.

\section{Conclusion}

In this study we assessed the association of SLC22A4 gene polymorphism rs3792876 with Chinese Han GD, HT and AITD patients, our results suggested lack of association between rs3792876 and GD, HT and AITD in the Chinese Han population.

\section{Competing interests}

The authors declare that they have no competing interests.

\section{Authors' contributions}

$\mathrm{XH}$ contributed to the collection, analysis of the clinical and genetic data on the subjects, and manuscript preparation. JM contributed to the analysis of the SNP results. JL and WW contributed to the collection of the clinical data on the subjects. CF, HW and $\mathrm{HZ}$ contributed to the biochemical measurements. WT, ZS and YL coordinated the study and helped to draft the manuscript. All authors read and approved the final manuscript.

\section{Acknowledgments}

We are grateful to all the patients and their pedigree members who participated in this study and the physicians who referred pedigrees and verified diagnoses. The study was supported by a grant from the National Natural Science Foundation of China (Grant: 30670996).

We acknowledge the help of Dr. Ying Jin who are from the Human Medical Genetics Program.

\section{Author details}

${ }^{1}$ Department of Endocrinology and Metabolism, The First Hospital of China Medical University, Key Laboratory of Endocrine Diseases, Liaoning Province 110001 Shenyang, China. ${ }^{2}$ Key Laboratory of Endocrine Diseases, Liaoning Province, Shenyang, China. ${ }^{3}$ Department of Geriatric Endocrinology and Metabolism, The First Hospital of China medical University, Shenyang, China.

Received: 28 December 2014 Accepted: 20 August 2015

Published online: 02 September 2015

\section{References}

1. Caturegli P, Kimura H, Rocchi R, Rose NR. Autoimmune thyroid diseases. Curr Opin Rheumatol. 2007;19(1):44-8.

2. Ban Y. Genetic factors of autoimmune thyroid diseases in Japanese. Autoimmune Dis. 2012. doi:10.1155/2012/236981.

3. Davies TF, Latif $R$, Yin X. New genetic insights from autoimmune thyroid disease. J Thyroid Res. 2012. doi:10.1155/2012/623852.

4. Bech K, Lumholtz B, Nerup J, Thomsen M, Platz P, Ryder LP, et al. HLA antigens in Graves' disease. Acta Endocrinol (Copenh). 1977:86(3):510-6.

5. Hadj-Kacem H, Rebuffat S, Mnif-Féki M, Belguith-Maalej S, Ayadi H, Péraldi-Roux S. Autoimmune thyroid diseases: genetic susceptibility of thyroid-specific genes and thyroid autoantigens contributions. Int J Immunogenet. 2009;36(2):85-96.

6. Stathatos N, Daniels GH. Autoimmune thyroid disease. Curr Opin Rheumatol. 2012;24(1):70-5.

7. Du L, Yang J, Huang J, Ma Y, Wang H, Xiong T, et al. The associations between the polymorphisms in the CTLA-4 gene and the risk of Graves' disease in the Chinese population. BMC Med Genet. 2013. doi:10.1186/1471-2350-14-46.

8. Jin Y, Teng W, Ben S, Xiong X, Zhang J, Xu S, et al. Genome-wide scan of Graves' disease: evidence for linkage on chromosome 5 q31 in Chinese Han pedigrees. J Clin Endocrinol Metab. 2003;88:1798-803.

9. Sakai K, Shirasawa S, Ishikawa N, Ito K, Tamai H, Kuma K, et al. Identification of susceptibility loci for autoimmune thyroid disease to 5q31-q33 and Hashimoto's thyroiditis to8q23-q24 by multipoint affected sib-pair linkage analysis in Japanese. Hum Mol Genet. 2001;10:1379-86.

10. Zhu W, Liu N, Zhao Y, Jia H, Cui B, Ning G. Association analysis of polymorphisms in IL-3, IL-4, IL-5, IL-9 and IL-13with Graves' disease. J Endocrinol Invest. 2010. doi:10.3275/6937.

11. Sharma V, Michel S, Gaertner V, Franke A, Vogelberg C, von Berg A, et al. Fine-mapping of IgE-associated loci 1q23, 5q31, and 12q13 using 1000 Genomes Project data. Allergy. 2014. doi:10.1111/all.12431.

12. Tokuhiro S, Yamada R, Chang X, Suzuki A, Kochi $Y$, Sawada T, et al. An intronic SNP in a RUNX1 binding site of SLC22A4, encoding an organic cation transporter, is associated with rheumatoid arthritis. Nat Genet. 2003;35:341-8

13. Santiago JL, Martínez A, de la Calle H, Fernández-Arquero M, Figueredo MA, de la Concha EG, et al. Evidence for the association of the SLC22A4 and SLC22A5 genes with Type 1 Diabetes: a case control study. BMC Medical Genetics. 2006. doi:10.1186/1471-2350-7-54.

14. Peltekova VD, Wintle RF, Rubin LA, Amos Cl, Huang Q, Gu X, et al. Functional variants of OCTN cation transporter genes are associated with Crohn disease. Nat Genet. 2004;36:471-5.

15. Helms C, Cao L, Krueger JG, Wijsman EM, Chamian F, Gordon D, et al. A putative RUNX1 binding site variant between SLC9A3R1 and NAT9 is associated with susceptibility to psoriasis. Nat Genet. 2003;35:349-56.

16. Prokunina L, Castillejo-López C, Oberg F, Gunnarsson I, Berg L, Magnusson $V$, et al. A regulatory polymorphism in PDCD1 is associated with susceptibility to systemic lupus erythematosus in humans. Nat Genet. 2002;32:666-9.

17. Barrett JC, Fry B, Maller J, Daly MJ. Haploview: analysis and visualization of LD and haplotype maps. Bioinformatics. 2005;21(2):263-5.

18. Rabinowitz D, Laird N. A unified approach to adjusting association tests for population admixture with arbitrary pedigree structure and arbitrary missing marker information. Human Hered. 2000;50:211-23.

19. $Y u$ Y, Sun LL, Jin Y, Li YS, Shan ZY, Huang W, et al. Association study between the IL4, IL13, IRF1 and UGRP1 genes in chromosomal 5 q31 region and Chinese Graves' disease. J Hum Genet. 2005;50:574-82.

20. Takata Y, Inoue H, Sato A, Tsugawa K, Miyatake K, Hamada D, et al. Replication of reported genetic associations of PADI4, FCRL3, SLC22A4 and RUNX1 genes with rheumatoid arthritis: results of an independent Japanese population and evidence from meta-analysis of East Asian studies. J Hum Genet. 2008;53(2):163-73. 
21. Okada Y, Mori M, Yamada R, Suzuki A, Kobayashi K, Kubo M, et al. SLC22A4 polymorphism and rheumatoid arthritis susceptibility: a replication study in a Japanese population and a meta analysis. J Rheumatol. 2008;35(9):1723-8.

22. Barton A, Eyre S, Bowes J, Ho P, John S, Worthington J. Investigation of the SLC22A4 Gene (Associated With Rheumatoid Arthritis in a Japanese Population) in a United Kingdom Population of Rheumatoid Arthritis Patients. Arthritis Rheum. 2005;52:752-8.

23. Martínez A, Valdivia A, Pascual-Salcedo D, Balsa A, Fernández-Gutiérrez B, De la Concha E, et al. Role of SLC22A4, SLC22A5, and RUNX1 genes in rheumatoid arthritis. J Rheumatol. 2006;33(5):842-6.

24. de Ridder L, Weersma RK, Dijkstra G, van der Steege G, Benninga MA, Nolte IM, et al. Genetic susceptibility has a more important role in pediatric-onset Crohn's disease than in adult-onset Crohn's disease. Inflamm Bowel Dis. 2006;13(9):1083-92.

\section{Submit your next manuscript to BioMed Central and take full advantage of:}

- Convenient online submission

- Thorough peer review

- No space constraints or color figure charges

- Immediate publication on acceptance

- Inclusion in PubMed, CAS, Scopus and Google Scholar

- Research which is freely available for redistribution 